\title{
Eigenvalue problems for Beltrami fields arising in a three-dimensional toroidal magnetohydrodynamic equilibrium problem
}

\author{
S. R. Hudson ${ }^{\text {a) }}$ \\ Princeton Plasma Physics Laboratory, P.O. Box 451, Princeton, New Jersey 08543 \\ M. J. Hole and R. L. Dewar \\ Department of Theoretical Physics and Plasma Research Laboratory, Research School of Physical Sciences \\ and Engineering, The Australian National University, ACT 0200 Australia
}

(Received 20 September 2006; accepted 15 March 2007; published online 11 May 2007)

\begin{abstract}
A generalized energy principle for finite-pressure, toroidal magnetohydrodynamic (MHD) equilibria in general three-dimensional configurations is proposed. The full set of ideal-MHD constraints is applied only on a discrete set of toroidal magnetic surfaces (invariant tori), which act as barriers against leakage of magnetic flux, helicity, and pressure through chaotic field-line transport. It is argued that a necessary condition for such invariant tori to exist is that they have fixed, irrational rotational transforms. In the toroidal domains bounded by these surfaces, full Taylor relaxation is assumed, thus leading to Beltrami fields $\nabla \times \mathbf{B}=\lambda \mathbf{B}$, where $\lambda$ is constant within each domain. Two distinct eigenvalue problems for $\lambda$ arise in this formulation, depending on whether fluxes and helicity are fixed, or boundary rotational transforms. These are studied in cylindrical geometry and in a three-dimensional toroidal region of annular cross section. In the latter case, an application of a residue criterion is used to determine the threshold for connected chaos. (C) 2007 American Institute of Physics. [DOI: 10.1063/1.2722721]
\end{abstract}

\section{INTRODUCTION}

The first step in the theoretical analysis of a toroidally confined plasma typically is to solve the equilibrium problem $\nabla p=\mathbf{j} \times \mathbf{B}$, where $p$ is the total plasma pressure (which we assume is scalar), $\mathbf{B}$ is the magnetic field, and $\mathbf{j}=\nabla \times \mathbf{B}$ is the plasma current density (using units such that the permeability of free space is unity), all quantities being implicitly functions of position $\mathbf{r}$. By dotting with $\mathbf{B}$ we find $\mathbf{B} \cdot \nabla p=0$. That is, $p$ is constant on a magnetic field line, transport in this direction being instantaneous on the MHD equilibrium time scale (i.e., much longer than wave propagation times).

In this paper we work within the general framework of magnetohydrodynamics (MHD), but we go beyond ideal MHD by supposing that the pointwise constraints of ideal MHD, such as the frozen-in flux condition, are relaxed over finite regions of the plasma; however, as in ideal MHD, we assume that the ion gyroradius is negligible compared with relevant scale lengths, so that the pressure is not necessarily a smooth function of position. The equilibria we seek must be consistent with ideal MHD, but also with the generalized relaxation model to be described below.

Toroidal plasmas are intimately connected to the magnetic fields they are embedded in, and toroidal magnetic field lines can be viewed, in a three-dimensional (3D) generalized coordinate space, as orbits obeying $1 \frac{1}{2}$-dimensional Hamiltonian dynamics. ${ }^{1-3}$ The $3 \mathrm{D}$ configuration space is regarded as a two-dimensional (2D) phase space extended by a "time" coordinate, which we take to be a suitably chosen generalized toroidal angle, $\zeta$. Thus a theory of 3D equilibrium must

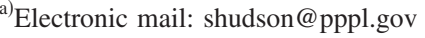

be a marriage of MHD theory and the Hamiltonian dynamics of magnetic field lines.

For systems with a continuous symmetry, e.g., axisymmetric systems, the time-like symmetry coordinate $\zeta$ may be chosen so the field-line Hamiltonian is autonomous. ${ }^{4}$ Then the Hamiltonian dynamics is integrable and describable in action-angle coordinates. ${ }^{5}$ The $2 \mathrm{D}$ phase space is foliated by invariant circles, corresponding in 3-space to foliation by nested toroidal magnetic flux surfaces. Each flux surface is characterized by its rotational transform, $t$, being the fieldline average with respect to $\zeta$ of the rate of poloidal rotation, $\partial \theta / \partial \zeta, \theta$ being a generalized poloidal angle. (In tokamaks the inverse, $q \equiv 1 / t$ is normally used.) When $t$ is rational, the field lines on the magnetic flux surface all close on themselves and the surface (really a family of periodic orbits) is referred to as a rational surface. When $t$ is irrational, a single field line ergodically covers the flux surface, and the surface is referred to as an irrational surface.

The condition $\mathbf{B} \cdot \nabla p=0$ implies that $p$ is constant on each magnetic surface and, as these smoothly foliate the plasma volume in the integrable case, the pressure profile may be taken to be a smooth function of position. The equilibrium problem is then well posed-one solves the GradShafranov equation, a nonlinear elliptic partial-differentialequation (PDE) for the magnetic flux function.

However for systems without a continuous symmetry, such as the necessarily three-dimensional stellarator, ${ }^{6}$ the construction of an equilibrium is much more subtle. The field-line Hamiltonian now depends on $\zeta$, and such nonautonomous, periodically forced, Hamiltonian systems generically exhibit partially chaotic behavior (see, for example, the dynamical systems texts by Arrowsmith and Place, ${ }^{7}$ and Lichtenberg and Lieberman, ${ }^{8}$ or the review article by 
Meiss $\left.{ }^{9}\right)$. This is the first problem we need to face.

Breaking of continuous symmetry destroys the rational surfaces through the formation of magnetic islands, and the chaotic tangles of the hyperbolic periodic orbits in the island chains appear to fill finite volumes ergodically. Thus, by virtue of $\mathbf{B} \cdot \nabla p=0$, such volumes must have constant pressure-plasma confinement is lost within these chaotic regions.

However, the Kolmogorov-Arnol'd-Moser (KAM) theorem (see, for example, Ref. 7, p. 330 or Ref. 8, p. 174) shows that a positive measure of invariant tori can survive small symmetry-breaking perturbations, provided the rotational transform $t$ is an irrational number obeying a Diophantine condition $|t-n / m| \geqslant c|m|^{-5 / 2}$ for some constant $c$ and all integers $m$ and $n$. Numerical approaches that complement the KAM theorem, such as Greene's residue criterion, ${ }^{10}$ show that a large measure of invariant tori can survive even strong symmetry breaking, and careful stellarator design seeks to maximize the volume of such "good flux surfaces" 11 as these separate the chaotic regions and thus act as barriers to chaotic transport. We shall assume some irrational magnetic surfaces survive and will call them KAM surfaces, whether or not the system is close to axisymmetry.

Not all surviving KAM surfaces are equal, in the sense that some are more robust to chaos than others. The KAM surfaces that are most robust are typically in regions furthest away from islands and have noble rotational transform..$^{10,12}$ (Noble irrational numbers are those that have an infinite tail of 1's in their continued-fraction representation. ${ }^{13}$ ) How close a given irrational surface is to destruction can be quantified by calculation of the residues of its rational convergents; ${ }^{10}$ the further the surface is from destruction, the more quickly the residues of the convergents approach zero. ${ }^{14}$ The KAM surfaces that lie adjacent to connected chaotic regions are called boundary surfaces. ${ }^{12,15}$ These boundary surfaces are critical, in the sense that they will be destroyed by a small increase in the chaos.

A popular approach to solving the equilibrium problem in nonaxisymmetric systems, embodied in the VMEC code, ${ }^{16}$ is to ignore the above comments about the genericity of field-line chaos and to proceed on the assumption that, even in nonaxisymmetric systems, the plasma volume is foliated with toroidal magnetic surfaces. Although this approach works remarkably well as a practical approximation, there are inherent problems at rational surfaces and careful convergence studies prove not to be possible. ${ }^{17}$ This is the second problem with nonaxisymmetric equilibria: pressure gradients are inconsistent with rational surfaces due to a singularity in the resonant component of the parallel current. ${ }^{18,19}$

However, pressure gradients are allowed at the irrational, and therefore nonresonant, KAM surfaces. This, and the fact that rationals are dense on the real line, requires us to give up the usual smoothness assumption for the pressure profile. Such a conclusion was reached by Grad, ${ }^{20}$ who concluded that only equilibria with "pathological nonsmooth pressure gradients" can be found.

At every level of detail, the field is a mix of ergodic field lines and periodic orbits, interspersed with KAM surfaces and cantori. One cannot hope to resolve the exact intricate structure of the field with finite numerical resolution, and must choose a model that approximates reality. For the ultimate success of any algorithm, the model must be mathematically self-consistent, with both the framework of MHD and with the chaotic structure of the magnetic field.

Two numerical approaches to calculating nonaxisymmetric equilibria that go beyond the VMEC assumption of foliation by magnetic surfaces have previously been developedPIES (Ref. 21) and HINT. ${ }^{22}$ These codes are both based on iterative procedures that may not lead to a unique answer if convergence studies, including mesh refinement, are done. At any rate, these codes are very numerically intensive and careful convergence studies are difficult. Thus we believe there is a need for a new approach in which there is a reasonably sound mathematical basis for believing that a unique solution exists, with a payoff in computational efficiency.

Our approach, described in Sec. II, is based on a generalization of the Kruskal-Kulsrud ${ }^{23}$ variational principle for MHD equilibria to incorporate partial Taylor relaxation. ${ }^{24,25}$ Regarding the pressure profile, all we require is that the volume integral of $p$ exists, so $p$ can be a discontinuous function of position. Thus, the variational approach provides a weak form of the ideal MHD equilibrium equation, allowing sheet currents to flow at a set of suitably chosen toroidal interfaces, where the pressure is discontinuous. By basing the method on a variational principle it is hoped that convergence to a unique equilibrium may be provable, though we do not attempt this here.

We have in mind a multiple-interface, sharp-boundary model. The pressure profile is piecewise-constant, with pressure jumps at a discrete set of selected KAM surfaces, which we call KAM barriers. (These ideal MHD barriers are not necessarily related to the transport barriers observed in experiments, and are related to, but distinct from, the cantorus transport barriers discussed by Misguich et al. ${ }^{26,27}$ ) This model is motivated by the realization that constant-pressure regions will be produced by chaotic field lines associated with unstable periodic orbits, and by setting $\nabla p=0$ across the island regions the model eliminates the problematic singularity in the pressure-driven parallel currents. The model does not require a family of continuously nested flux surfaces; the only flux surfaces required are those whose existence is provided by the KAM theorem and its extensions.

In the context of constructing a general-purpose global equilibrium code, the pressure jumps would be chosen to approximate some desired profile. The closer to integrable the magnetic field is, the more KAM surfaces exist, the more pressure jumps may be used, and the smaller they may be made-little physical generality is really lost by giving up differentiability of the pressure profile.

A similar discontinuous pressure model is considered by Bruno and Laurence, ${ }^{28}$ who showed analytically that this model allows solutions to the ideal equilibrium equations for sufficiently simple, but nonaxisymmetric, toroidal geometry. An earlier treatment of ideal MHD equilibria, in 3D geometry, where a sharp boundary separates a uniform pressure plasma from a vacuum, is described by Berk et al. ${ }^{29}$ Kaiser and Salat ${ }^{30}$ showed that sharp-boundary equilibria, with suf- 
ficiently irrational rotational transform, exist for configurations with small deviations from axisymmetry, and Kaiser ${ }^{31}$ showed that for every rational-rotational-transform boundary, an arbitrarily weak deformation exists such that the equilibrium is destroyed. Relaxed plasma-vacuum systems are also considered by Spies and co-workers. ${ }^{32-34}$

The analytical results demonstrating existence of sharpboundary equilibria are a strong motivation to pursue this model further. The analytical results are however restricted to small deviations from axisymmetry. The need to treat strongly nonaxisymmetric systems dictates that we must be prepared to treat the problem numerically, and this paper is a preliminary scoping study of a new approach to doing this.

We have not yet implemented the above ideas numerically to obtain a global equilibrium code, but this article discusses a number of relevant questions for such a model. First, a generalized, relaxed energy principle that incorporates elements of ideal MHD, partial Taylor relaxation and chaotic Hamiltonian theory is sketched, Sec. II. By setting the first variation of the energy to zero, this model implies that the magnetic field in a subregion $i$, where the pressure is flat, obeys $\nabla \times \mathbf{B}=\lambda_{i} \mathbf{B}$, i.e. it is a Beltrami field.

However, the variational principle as it stands does not constrain the rotational transforms at the boundaries of the subdomains to be fixed. As the preceding discussion suggests this would be highly desirable. The nature of the boundary value problem posed by solving the Beltrami equation within a toroidal domain with rotational transform(s) specified on the boundary(ies) is examined in cylindrical geometry, Sec. III, where the field is given by Bessel functions.

To extend this to $3 \mathrm{D}$, a numerical method for constructing Beltrami fields in generally shaped toroidal regions of annular cross section is presented in Sec. IV. As an illustration, the method is applied, Sec. V, to a nonaxisymmetric system with a perturbed outer boundary. Such fields are, in general, partially chaotic; so an analysis of the chaotic field based on the residue criterion ${ }^{10}$ is also presented.

This analysis allows the degree of chaos to be quantified, and suggests which KAM surfaces exist for a given level of perturbation. Such KAM surfaces could be used as additional interfaces to further subdivide the region, and some comments on this are given in Sec. VI. The multiple interface problem with a stepped pressure profile in arbitrary threedimensional geometry is, however, left for future work.

\section{PARTIAL TAYLOR RELAXATION}

Our proposed generalization of the Kruskal-Kulsrud ${ }^{23}$ variational principle is similar to that of Bhattacharjee and Dewar, ${ }^{35}$ who extremize the total free energy

$$
F=W-\sum_{i} \lambda_{i} K_{i}-\sum_{i} \mu_{i} M_{i}-\sum_{i} T_{i} S_{i},
$$

where the total energy $W$ is defined by

$$
W \equiv \int_{V} d \tau\left[\frac{B^{2}}{2}+\frac{p}{\gamma-1}\right]
$$

with $V$ being the plasma volume (assuming, for simplicity, the plasma to be confined by a perfectly conducting shell) and $d \tau$ a volume element. Also, $\gamma$ is the ratio of specific heats, the plasma equation of state being assumed to be that of an ideal gas. The other quantities in Eq. (1) are defined below.

The minimization is subject to only a subset of the full set of ideal MHD continuum constraints assumed by Kruskal and Kulsrud. In particular, the constraint set includes a finite number of moments $K_{i} \equiv K\left[w_{i}\right]$, of $\mathbf{A} \cdot \mathbf{B}$ (A being a singlevalued vector potential for $\mathbf{B}$ ) with respect to weight functions $w_{i}(\mathbf{r})$ such that $\mathbf{B} \cdot \nabla w_{i}=0$ everywhere, so that any moment

$$
K\left[w_{i}\right] \equiv \frac{1}{2} \int_{V} d \tau w_{i} \mathbf{A} \cdot \mathbf{B}
$$

is invariant under ideal-MHD Eulerian variations $\delta \mathbf{A}=\boldsymbol{\xi}$ $\times \mathbf{B}+\nabla \delta \chi$, where $\boldsymbol{\xi}$ is an arbitrary infinitesimal fluid displacement (such variations satisfying the frozen-in flux condition). The arbitrary function $\delta \chi$ is to allow infinitesimal gauge transformations. We assume such gauge functions $\chi$ to be single-valued functions of $\mathbf{r}$, so that they do not affect loop integrals of $\mathbf{A}$ (which are physical fluxes). Then the contribution of $\chi$ to $K\left[w_{i}\right]$ vanishes by Gauss' theorem and the assumption that $\mathbf{B}$ is tangential to the boundary of $V$. That is, $K\left[w_{i}\right]$ is gauge-invariant as it stands and does not need the loop-integral corrections sometimes used. ${ }^{33}$

For $w_{i}=1, K$ is the magnetic helicity. Thus the Bhattacharjee-Dewar variational principle is a generalization of the variational principles of Woltjer ${ }^{36}$ and Taylor $^{24}$ for force-free equilibria.

Likewise, $M_{i} \equiv M\left[u_{i}\right]$ and $S_{i} \equiv S\left[v_{i}\right]$ are moments of the mass density $\rho$ and entropy density $\ln \left(p / \rho^{\gamma}\right) \rho /(\gamma-1)$ with respect to weight functions $u_{i}$ and $v_{i}$ chosen such that $M\left[u_{i}\right]$ and $S\left[v_{i}\right]$ are conserved under ideal variations $\delta \rho=-\nabla \cdot(\rho \xi)$, $\delta p=-\gamma p \nabla \cdot \boldsymbol{\xi}-\boldsymbol{\xi} \cdot \nabla p$. (As the mass is irrelevant for the equilibrium problem, it is possible to combine these two constraints into one $\mathrm{e}^{23}$ but it is clearer physically to keep them as separate constraints.)

In solving the resulting Euler-Lagrange equations, the Lagrange multipliers $\lambda_{i}, \mu_{i}$, and $T_{i}$ are, in principle, to be determined from the constraints: the moments chosen to be conserved should have fixed values. The method thus gives more control over profiles than allowed by Taylor's original relaxation idea (which was really suitable only for modeling strongly turbulent reversed-field pinches), but gives up the detailed control allowed by the original Kruskal-Kulsrud approach. By making the constraints a subset of the ideal-MHD invariants, the space of allowed variations is larger than that allowed by ideal MHD but includes it as a subspace. Thus the extremizing solutions of $F$ are automatically ideal-MHD equilibria, ${ }^{23}$ and minimization of $F$ provides a sufficient condition for $W$ to be minimal under ideal-MHD variations. (This approach is analogous to the Energy-Casimir method, ${ }^{37}$ often called Arnold's method.)

Bhattacharjee and Dewar ${ }^{35}$ assume the magnetic field to be integrable, so that $V$ is foliated by magnetic surfaces. They then take the weight functions to be smooth (polynomial) functions of the poloidal and toroidal flux functions, $\Psi^{p}$ and $\Psi^{t}$. As discussed in Sec. I, the assumption that mag- 
netic surfaces smoothly foliate the volume is not appropriate for nonaxisymmetric systems, so the smooth weight functions of Ref. 35 are not appropriate to the 3D equilibrium problem. Instead we propose to extremize a free energy $F$ given by Eq. (1), but to use unit hat-function weights with support confined to regions bounded by invariant tori of the magnetic field, which we shall call flux barriers $S_{i}$. This replaces the constant-global-helicity constraint of the original Taylor relaxation theory with multiple constraints of constant helicity in the local subregions, $V_{i}$, bounded by the surfaces $S_{i-1}$ and $S_{i}$.

Our picture of the flux barriers is that they are arbitrarily thin shells of ideal plasma, which the magnetic field cannot penetrate. This picture also implies the constraint that the magnetic field be tangential to the boundaries of the $V_{i}$,

$$
\mathbf{B} \cdot \mathbf{n}=0 \quad \text { on } S_{i},
$$

where the unit vector $\mathbf{n}$ is normal to $S_{i}$. This is a Lagrangian constraint in that, if the flux barrier is deformed, it applies on the modified surface. In the context of infinitesimal variations we distinguish Eulerian (fixed spatial position) variations $\delta$ from Lagrangian variations $\Delta \equiv \delta+\boldsymbol{\xi} \cdot \nabla$, where $\boldsymbol{\xi}(\mathbf{r})$ is an infinitesimal displacement field that carries $S_{i}$ from its original to its varied shape. Then the most general Eulerian variations in $\mathbf{A}$ that preserve $\Delta(\mathbf{n} \cdot \mathbf{B})=0$ as a Lagrangian invariant are

$$
\delta \mathbf{A}=\boldsymbol{\xi} \times \mathbf{B}+\delta a \mathbf{n}+\nabla \delta \chi \quad \text { on } S_{i},
$$

where $\delta a$ is an arbitrary function that allows nonideal variations and $\delta \chi$ is an arbitrary gauge term. This constraint leaves loop integrals of $\mathbf{A}$ as Lagrangian invariantsmagnetic fluxes are conserved-and also conserves the helicities $K_{i}$ within the subregions between the flux barriers.

The Euler-Lagrange equations resulting from extremizing the functional $F$, with weight functions $u_{i}=v_{i}=w_{i}=1$ in each subdomain $V_{i}$ give [see Eqs. (96) and (97) of Ref. 35] $\nabla p_{i}=0$ and the Beltrami equations

$$
\nabla \times \mathbf{B}=\lambda_{i} \mathbf{B} .
$$

Varying the boundaries $S_{i}[\boldsymbol{\xi} \neq 0$ in Eq. (5)] gives another Euler-Lagrange equation, the continuity of the total pressure across each flux barrier:

$$
\left[\left[p+\frac{1}{2} B^{2}\right]\right]=0
$$

where $[[\cdot]]$ denotes a jump. Pressure jumps (and rotational transform jumps) are allowed at the interfaces, $[[p]]=$ $-\left[\left[\frac{1}{2} B^{2}\right]\right]$, and thus a nontrivial pressure profile can be constructed by connecting together multiple subregions with different pressure.

If we imagine the flux barriers broadened into finitewidth shells of ideal plasma, retaining the full set of idealMHD constraints, we might argue that rotational transforms $t$ on the boundaries of these barriers (or throughout the shells, if they have zero magnetic shear) are "frozen in," and cannot change even if the shape of $S_{i}$ is varied. While this is true within the ideal plasma shell, the excitation of skin currents at the boundaries to exclude flux penetration from the relaxation regions means that the rotational transforms at the boundaries of the relaxation regions, just outside the ideal shells, are not necessarily fixed during arbitrary variations of the $S_{i}$.

Thus our variational principle does not constrain rotational transforms at the boundaries (as is further discussed in the next section). However, as was argued in Sec. I on the basis of KAM theory, supported by the result of Kaiser ${ }^{31}$ (Theorem 1), we believe it to be necessary for these $t$ to be irrational numbers in order for the invariant tori $S_{i}$ to exist. That is, we need to identify the flux barriers with KAM barriers. In the present variational formulation at least, the selection of suitable irrational $t$ must be imposed after the variation of $F$, as a further optimization step. As part of this process we need to consider the solution of Eq. (6) in each region $V_{i}$ under the combined homogeneous boundary conditions Eq. (4), and prescribed boundary rotational transforms. As discussed further in Sec. III, this provides an eigenvalue equation for $\lambda_{i}$ that is distinct from the eigenvalue problem implied by flux and helicity conservation.

\section{EIGENVALUE PROBLEMS IN A CYLINDER}

In this section we examine the problem of solving Eq. (6) in an annular toroidal subdomain $V_{i}$ under the condition of tangential $\mathbf{B}$ at the boundary, plus other appropriate boundary or other conditions. We use a geometry that is sufficiently simple that the magnetic field is integrable and existence and uniqueness can be examined by explicit construction rather than rigorous formal proof-a circular cylinder, with periodic boundary conditions to make it topologically toroidal. Note, taking the curl of Eq. (6), that we are dealing with a wave equation, $\nabla^{2} \mathbf{B}+\lambda^{2} \mathbf{B}=0$.

Consider the conditions implied by the variational principle proposed in the previous section, imagining that $F$ is first extremized with respect to variations interior to the subdomain $V_{i}$ [giving Eq. (6)], then with respect to deformations of the inner and outer flux barriers $S_{i-1}$ and $S_{i}$, respectively. Equation (5) implies that the appropriate conditions under which to solve Eq. (6) are fixed toroidal and poloidal fluxes, $\Psi^{t}$ and $\Psi^{p}$, plus fixed helicity $K_{i}$. Because Eq. (6) is linear, the overall magnitude of the magnetic field is arbitrary. Thus, without loss of generality, we can reduce the number of conditions to two by normalizing with respect to $\Psi^{t}$, so the pair of homogeneous conditions that need to be specified is $\left(\Psi^{p} / \Psi^{t}, K_{i} / \Psi^{t 2}\right)$. This can only be satisfied for special values of $\lambda_{i}$-it is an eigenvalue problem.

As argued previously, we also wish to specify the rotational transforms, $\boldsymbol{t}_{i-1}$ and $\boldsymbol{t}_{i}$, on the inner and outer bounding surfaces of $V_{i}$. These boundary conditions do not specify the strength of the magnetic field, forming a pair of homogeneous boundary conditions, thus also posing an eigenvalue problem. Are these two eigenvalue problems equivalent or different? After deriving expressions for the rotational transform, and the fluxes and helicity, we conclude that these problems are distinct. 
There are at least three other such eigenvalue problems in the Beltrami equation literature, defined by different homogeneous boundary conditions-Kress ${ }^{38}$ (Theorem 2) poses the problem of orthogonality, in terms of an inner product defined as a boundary integral, to all Neumann fields allowed in the complementary domain; Kress ${ }^{39}$ [Eqs. (3.35)(3.36)] poses an eigenvalue problem for a scalar field with vanishing Dirichlet boundary condition; and Kress ${ }^{40}$ (Theorem 2.5) shows that there exists a countable set of eigenvalues, accumulating only at infinity, in the case of requiring zero toroidal flux. The two problems posed above are presumably distinct from any of these.

The equilibrium of single-interface plasma-vacuum cylindrical systems has been solved by Lortz and Spies ${ }^{41}$ (for zero pressure) and Kaiser and Uecker ${ }^{42}$ (for nonzero pressure). Recently, Hole et $a l^{43}$ extended the analysis to multiple-interface configurations, and studied the stability of such configurations. ${ }^{44}$

In cylindrical coordinates $(r, \theta, z)$, solutions for the magnetic field $\mathbf{B}=\left\{B_{r}(r), B_{\theta}(r), B_{z}(r)\right\}$ between two interfaces in the plasma can be written

$$
\left(\begin{array}{c}
B_{r} \\
B_{\theta} \\
B_{\zeta}
\end{array}\right)=\left(\begin{array}{c}
0 \\
\operatorname{sgn}\left(\lambda_{i}\right)\left[k_{i} J_{1}\left(\left|\lambda_{i}\right| r\right)+d_{i} Y_{1}\left(\left|\lambda_{i}\right| r\right)\right] \\
k_{i} J_{0}\left(\left|\lambda_{i}\right| r\right)+d_{i} Y_{0}\left(\left|\lambda_{i}\right| r\right)
\end{array}\right),
$$

where $k_{i}, d_{i} \in \mathbb{R}$, and $J_{0}, J_{1}$ and $Y_{0}, Y_{1}$ are Bessel functions of the first kind of order 0,1 , and second kind of order 0,1 , respectively. The coefficients $k_{i}$ and $d_{i}$, which are constant in the subvolume $V_{i}$, are solved using the interface condition $\left[\left[p+\frac{1}{2} B^{2}\right]\right]=0$ and an auxiliary constraint such as the rotational transform at either interface. The cylinder is assumed to be axially periodic with length $L$.

In the cylinder, the rotational transform at radius $r$ is

$$
\boldsymbol{t}=\frac{L}{2 \pi r} \frac{B_{\theta}(r)}{B_{z}(r)} .
$$

Consider a plasma region with Lagrange multiplier $\lambda_{2}$ bounded by two cylindrical interfaces, $r_{1}$ and $r_{2}$, with $r_{1}$ $<r_{2}$. Also, let us assume that the rotational transform immediately outside the inner interface $r_{1}$ is prescribed, with the value $t_{1}$. Substituting for the field Eq. (8) gives the expression

$$
\boldsymbol{t}_{1}=\operatorname{sgn}\left(\lambda_{2}\right) \frac{L}{2 \pi r_{1}} \frac{J_{1}\left(\left|\lambda_{2}\right| r_{1}\right)+\left(d_{2} / k_{2}\right) Y_{1}\left(\left|\lambda_{2}\right| r_{1}\right)}{J_{0}\left(\left|\lambda_{2}\right| r_{1}\right)+\left(d_{2} / k_{2}\right) Y_{0}\left(\left|\lambda_{2}\right| r_{1}\right)}
$$

which can be solved for the ratio $d_{2} / k_{2}$, giving

$$
\frac{d_{2}}{k_{2}}=\frac{J_{0}\left(\left|\lambda_{2}\right| r_{1}\right)-\frac{\operatorname{sgn}\left(\lambda_{2}\right) L}{2 \pi r_{1} t_{1}} J_{1}\left(\left|\lambda_{2}\right| r_{1}\right)}{-Y_{0}\left(\left|\lambda_{2}\right| r_{1}\right)+\frac{\operatorname{sgn}\left(\lambda_{2}\right) L}{2 \pi r_{1} t_{1}} Y_{1}\left(\left|\lambda_{2}\right| r_{1}\right)} .
$$

Substituting Eq. (11) into Eq. (9) evaluated at interface $r_{2}$ gives an expression for $t_{2}$, the rotational transform immediately inside the outer interface, as a function of $L, r_{1}, r_{2}, t_{1}$ and $\lambda_{2}$, which can in principle be inverted to give $\lambda_{2}$ as a

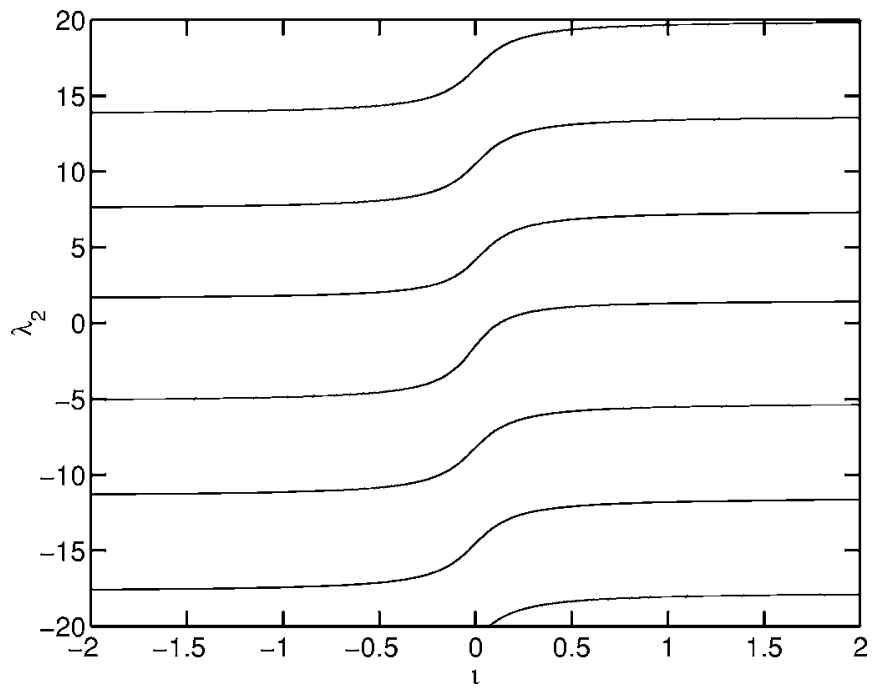

FIG. 1. Eigenvalues $\lambda_{2}$ for cylindrical plasma as a function of $t_{2}$, with $r_{1}$ $=0.5, r_{2}=1$, and $t_{1}=0.5$.

function of $t_{2}$ (but not a unique function as we shall now see).

Figure 1 shows the eigenvalues, $\lambda_{2}$, as a function of $t_{2}$, for $r_{1}=0.5, r_{2}=1$, and $t_{1}=0.5$. For any given $t_{2}$, solutions are multivalued - the solution is not unique unless the solution branch is specified. The solution branch with the lowest $\lambda_{2}$, the "fundamental," corresponds to a continuous $t$ profile between $r_{1}$ and $r_{2}$. Branches corresponding to higher "overtones" are separated by poles in the rotational transform profile, where the axial field passes through zero. The $n$th overtone passes $n$ times through $t=\infty$, in each instance generating a field reversal. Figure 2 shows the $t$ profile as a function of radius between the two interfaces for the fundamental and first two $\lambda_{2}>0$ overtones. The fundamental

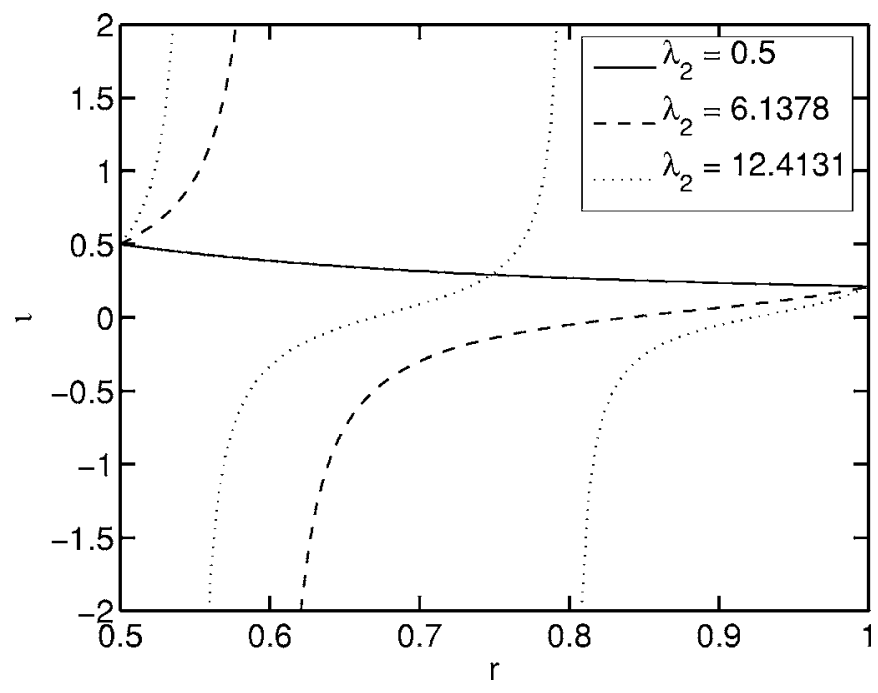

FIG. 2. Rotational transform profile for the first three eigenvalues with $r_{1}$ $=0.5, r_{2}=1, t_{1}=0.5$, and $t_{2}=0.212$. 


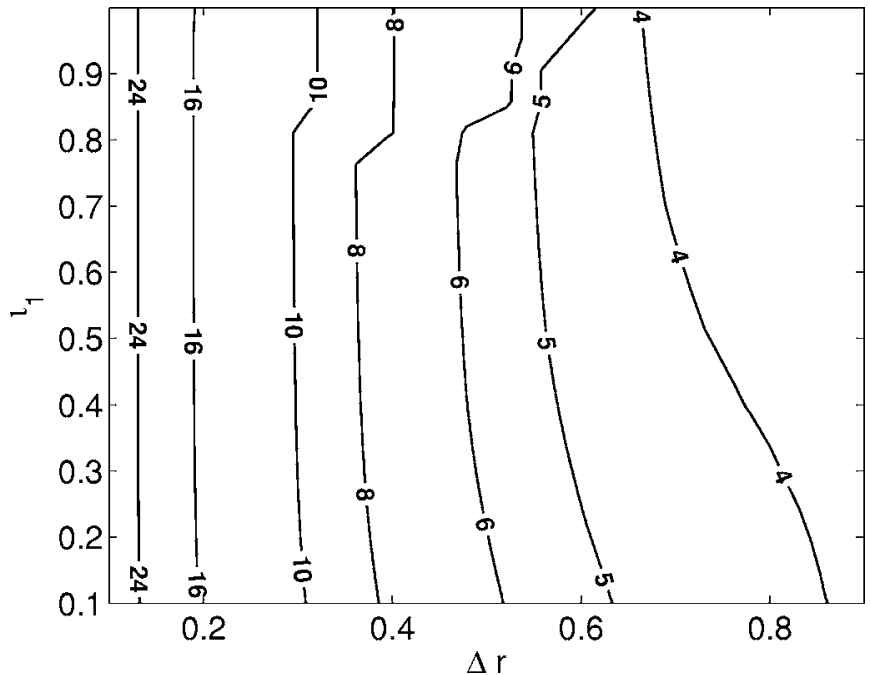

FIG. 3. Contour plot of spacing between lowest eigenvalues $\Delta \lambda_{2}$ as a function of interface separation $\Delta r=r_{2}-r_{1}$ and $t_{1}$. The contour labels display the value of $\Delta \lambda$.

varies continuously, while the first and second overtones have field reversals at $r=0.60$, and $r=0.55$ and $r=0.80$, respectively. Overtones with $\lambda_{2}<0$ have opposite shear to those with $\lambda_{2}>0$.

The eigenvalue spacing, $\Delta \lambda_{2}$, for common values of $t_{2}$ in the cylindrical case is also of interest, as it provides a useful guide for solution searches in more complicated geometry. Figure 3 is a contour plot of the eigenvalue spacing between the lowest two $\lambda_{2}>0$ eigenvalues as a function $t_{1}$ and interface separation $\Delta r=r_{2}-r_{1}$. As the interface separation decreases, the eigenvalue spacing increases, and the Lagrange multiplier must increase to supply the shear required to match any difference in rotational transform; this increases the fundamental and the spacing between overtones. For large interface separation, the eigenvalue spacing increases weakly with decreasing $\boldsymbol{t}_{1}$. For $\boldsymbol{t}_{1} \ll 1$, Eq. (11) gives $d_{2} / k_{2}$ $\approx-J_{1}\left(\left|\lambda_{2}\right| r_{1}\right) / Y_{1}\left(\left|\lambda_{2}\right| r_{1}\right)$, and so $t_{2} \gg 1$. Consequently, both the fundamental and $\Delta \lambda_{2}$ must increase to match the change in $t$. Finally, we also note that the spacing is not identical between higher overtones, due to the nonuniform spacing of zeros of the Bessel functions.

The poloidal and toroidal flux in the region between $r_{1}$ and $r_{2}$ is given by

$$
\begin{aligned}
& \Psi_{2}^{t}=\int_{r_{1}}^{r_{2}} B_{z}(r) r d \theta d r=\frac{2 \pi}{\left|\lambda_{2}\right|}\left[k_{2} r J_{1}\left(r\left|\lambda_{2}\right|\right)+d_{2} r Y_{1}\left(r\left|\lambda_{2}\right|\right)\right]_{r_{1}}^{r_{2}}, \\
& \Psi_{2}^{p}=\int_{r_{1}}^{r_{2}} B_{\theta}(r) L d r=\frac{-L}{\lambda_{2}}\left[k_{2} J_{0}\left(r\left|\lambda_{2}\right|\right)+d_{2} Y_{0}\left(r\left|\lambda_{2}\right|\right)\right]_{r_{1}}^{r_{2}},
\end{aligned}
$$

while the helicity is

$$
\begin{aligned}
& K_{2}=L \pi \int_{r_{1}}^{r_{2}} \lambda_{2}^{-1} B^{2} d r \\
& =\frac{L \pi}{2 \lambda_{2}}\left\{k_{2}^{2} r^{2}\left[J_{0}\left(r\left|\lambda_{2}\right|\right)^{2}+2 J_{1}\left(r\left|\lambda_{2}\right|\right)^{2}-J_{0}\left(r\left|\lambda_{2}\right|\right) J_{2}\left(r\left|\lambda_{2}\right|\right)\right]\right. \\
& +d_{2}^{2} r^{2}\left[Y_{0}\left(r\left|\lambda_{2}\right|\right)^{2}+2 Y_{1}\left(r\left|\lambda_{2}\right|\right)^{2}-Y_{0}\left(r\left|\lambda_{2}\right|\right) Y_{2}\left(r\left|\lambda_{2}\right|\right)\right] \\
& +\frac{2 d_{2} k_{2} r^{2}}{\sqrt{\pi}}\left[G_{2,4}^{2,1}\left(r\left|\lambda_{2}\right|, \frac{1}{2} \mid \begin{array}{c}
\frac{1}{2},-\frac{1}{2} \\
0,0,-1,-\frac{1}{2}
\end{array}\right)\right. \\
& \left.\left.+G_{3,5}^{2,2}\left(r\left|\lambda_{2}\right|, \frac{1}{2} \mid \begin{array}{c}
0, \frac{1}{2},-\frac{1}{2} \\
0,1,-1,-1,-\frac{1}{2}
\end{array}\right)\right]\right\}_{r_{1}}^{r_{2}}
\end{aligned}
$$

where $G$ is a generalized form of the Meijer $G$ function. ${ }^{45}$

Comparing Eqs. (12)-(15) with Eqs. (8)-(11) we see there can be no simple connection between the two eigenvalue problems (prescribed normalized helicity and flux, or prescribed rotational transforms). Note that for calculating the helicity we needed an expression for the vector potential A. The most general form of the vector potential is $\mathbf{A}$ $=\mathbf{B} / \lambda_{2}+\nabla \chi$ [the curl of this giving the Beltrami equation, Eq. (6)], the gauge function $\chi$ in general including secular terms $c^{p} \theta+c^{t} \zeta$, with the constants $c^{p}, c^{t}$ chosen to adjust the boundary loop integrals of $\mathbf{A}$ to their prescribed values. However, as we calculated $K_{2}$ simply to illustrate that the helicity bears no simple relation to the rotational transform, in Eq. (15) $\chi$ was taken to be zero.

In the cylindrical case, the simple geometry allows an analytic solution for the Beltrami field in each region. In arbitrary 3D geometry the solution must be found numerically, and a method for doing this is described in the following section.

\section{GENERAL 3D SOLUTION}

We now consider a single annular region bounded by two interfaces $S_{i}$ and $S_{i+1}$, whose geometry is assumed given. The pressure is constant in this region, with pressure jumps $\delta p_{i}, \delta p_{i+1}$ at the interfaces. We seek the field that satisfies $\nabla \times \mathbf{B}=\lambda \mathbf{B}$ for a given constant $\lambda$.

We obtain the solution by casting this equation, really a system of three PDEs, as a system of ordinary-differentialequations (ODEs) for the covariant components of the magnetic field.

We now set up a curvilinear coordinate system in the domain such that $\mathbf{r}=\mathbf{x}(s, \theta, \zeta), \mathbf{r}$ being the position vector $\mathbf{r}$ $=r \hat{r}+z \hat{z}$, where $\hat{r}$ and $\hat{z}$ are unit vectors in the $r$ and $z$ directions of an underlying cylindrical coordinate system. We take the inner and outer interfaces, $S_{i}: \mathbf{r}=\mathbf{x}_{i}(\theta, \zeta)$, and $S_{i+1}: \mathbf{r}$ $=\mathbf{x}_{i+1}(\theta, \zeta)$, to coincide with level surfaces of the coordinate $s$, where $\mathbf{x}_{j}=R_{j}(\theta, \zeta) \hat{r}+Z_{j}(\theta, \zeta) \hat{z}$, with $j=i$ or $i+1$. We take $\theta$ 
and $\zeta$ to be, respectively, poloidal and toroidal angle coordinates that may be chosen arbitrarily on the interfaces $S_{i}$ and $S_{i+1}$. Then, provided the domain is not too contorted, the coordinate system may be extended into the domain by linear interpolation, $\mathbf{x}=s \mathbf{x}_{i+1}+(1-s) \mathbf{x}_{i}$. Other than on the bounding interfaces, no assumption is made that the level surfaces of $s$ correspond to magnetic surfaces.

By writing the magnetic field in covariant form,

$$
\mathbf{B}=B_{s} \nabla s+B_{\theta} \nabla \theta+B_{\zeta} \nabla \zeta,
$$

the components of the equation $\nabla \times \mathbf{B}=\lambda \mathbf{B}$ are manipulated to isolate the partial radial derivatives of $B_{\theta}$ and $B_{\zeta}$

$$
\begin{aligned}
& \partial_{s} B_{\theta}=\partial_{\theta} B_{s}+\lambda \sqrt{g}\left(g^{s \zeta} B_{s}+g^{\theta \zeta} B_{\theta}+g^{\zeta \zeta} B_{\zeta}\right), \\
& \partial_{s} B_{\zeta}=\partial_{\zeta} B_{s}-\lambda \sqrt{g}\left(g^{s \theta} B_{s}+g^{\theta \theta} B_{\theta}+g^{\zeta \theta} B_{\zeta}\right),
\end{aligned}
$$

where the "raising" metric elements are $g^{i j}=\nabla u^{i} \cdot \nabla u^{j}$ for $\left(u^{1}, u^{2}, u^{3}\right)=(s, \theta, \zeta)$, and the notation $\partial_{u} f$ represents the partial derivative of $f$ with respect to the coordinate $u$.

We obtain equations determining $B^{s} \equiv \mathbf{B} \cdot \nabla s$, the component of the field normal to the coordinate surface, and $B_{s}$ in terms of $B_{\theta}, B_{\zeta}$ :

$$
B^{s}=\frac{\partial_{\theta} B_{\zeta}-\partial_{\zeta} B_{\theta}}{\lambda \sqrt{g}},
$$

and

$$
B_{s}=\frac{B^{s}-g^{s \theta} B_{\theta}-g^{s \zeta} B_{\zeta}}{g^{s s}} .
$$

The toroidal geometry suggests a Fourier representation. For stellarator-symmetric configurations, ${ }^{46}$ each interface is written

$$
\begin{aligned}
& R=\sum_{m, n} R_{m, n} \cos (m \theta-n \zeta), \\
& Z=\sum_{m, n} Z_{m, n} \sin (m \theta-n \zeta) .
\end{aligned}
$$

We adopt a VMEC-like convention, where for the $m=0$ Fourier harmonics, $n$ varies from 0 to $N$, and for $m=1$ to $M, n$ varies from $-N$ to $N$. This gives a total of $N_{m n}=(N+1)$ $+M(2 N+1)$ harmonics. For odd functions, the $(m, n)$ $=(0,0)$ harmonic is irrelevant, giving $N_{n m}-1$ relevant harmonics.

The covariant components of the field are written

$$
\begin{aligned}
& B_{\theta}=\sum_{m, n} B_{\theta, m, n}(s) \cos (m \theta-n \zeta), \\
& B_{\zeta}=\sum_{m, n} B_{\zeta, m, n}(s) \cos (m \theta-n \zeta),
\end{aligned}
$$

with $B_{s}$ being determined by Eq. (20). To be consistent with the Fourier representation, in solving for $B_{s}$ from Eq. (20) the right-hand side is Fourier decomposed (on a poloidal, toroidal grid with resolution $N_{j}, N_{k}$, with the terms outside the range determined by $N, M$ being truncated. We are thus left with $N_{m n}-1$ harmonics for the odd function $B_{s}$.
Within the Fourier representation the angular derivatives are trivial. The partial radial derivatives of the $B_{\theta}, B_{\zeta}$ become total radial derivatives for the Fourier harmonics

$$
\frac{d}{d s} B_{\theta, m, n}=-m B_{s, m, n}+\lambda\left[\sqrt{g}\left(g^{s \zeta} B_{s}+g^{\theta \zeta} B_{\theta}+g^{\zeta \zeta} B_{\zeta}\right)\right]_{m, n},
$$

$$
\frac{d}{d s} B_{\zeta, m, n}=n B_{s, m, n}-\lambda\left[\sqrt{g}\left(g^{s \theta} B_{s}+g^{\theta \theta} B_{\theta}+g^{\zeta \theta} B_{\zeta}\right)\right]_{m, n} .
$$

These equations can be integrated radially from the inner interface to the outer interface: all that is required is to provide an initial condition.

We must obtain initial values for the $B_{\theta, m, n}, B_{\zeta, m, n}$ such that (i) the field is tangential to the inner interface, and (ii) the field as obtained by integrating the system of ODEs is tangential to the outer interface. The first condition can be satisfied by construction, and the second condition can be determined iteratively, as will now be described.

The boundary condition at each interface is that $\mathbf{B} \cdot \mathbf{n}$ $=0$, where $\mathbf{n}$ is normal to the interface. By virtue of the coordinate construction, $\mathbf{n}=\nabla s /|\nabla s|$ at each interface, so this condition is $B^{s}=0$. Thus, by Eq. (19),

$$
\partial_{\theta} B_{\zeta}-\partial_{\zeta} B_{\theta}=0 .
$$

This can be satisfied by construction:

$$
B_{\theta}=\partial_{\theta} f, \quad B_{\zeta}=\partial_{\zeta} f
$$

for any function $f(\theta, \zeta)$. The most general form for $f$, consistent with toroidal periodicity and stellarator symmetry, is

$$
f=I \theta-G \zeta+\sum_{m, n} f_{m n} \sin (m \theta-n \zeta) .
$$

The quantities $I, G$ are directly determined by the toroidal and poloidal currents and, together with the description of the boundary, complete the specification of the mathematical problem. The rotational transform on the inner surface is determined by the ratio $I / G$, and the magnitude of the shear is related to $\lambda$. The function $f$, with $N_{m n}-1$ degrees of freedom, determines the tangential field at the inner interface. It is this freedom in the tangential field that allows one to construct a field that is also tangential to the outer interface, as described below.

Beginning with an initial guess for $f$, the system of ODEs can be integrated radially to obtain the solution within the domain. At the inner interface, $B^{s}=0$ by construction; but, generally $B^{s}$ will not remain zero as the integration proceeds, and the condition that the field be tangential to the outer interface will not be satisfied.

Within the Fourier representation, there are $N_{m n}-1$ constraints: each of the Fourier harmonics of $B^{s}$, as given by Eq. (27), must be zero at $s=1$. There exist as many constraints as degrees of freedom, and the solution may be determined iteratively. We have implemented a multidimensional Newton method and this approach allows Beltrami fields to be constructed in an annulus of arbitrary geometry. 
The system of ODEs is integrated from $s=0$ with an initial guess for $f_{m, n}$, typically $f_{m, n}=0$, using a Runge-Kutta algorithm (the NAG routine D02BJF). The derivatives of the "error-vector," $B_{m, n}^{s}$, at $s=1$, with respect to the independent variables $f_{m, n}$, are calculated (in parallel) using finitedifferences. In all the cases considered in the following section, the solution was obtained in 2-3 Newton iterations, to an error with magnitude $\sim 10^{-13}$. The cpu requirements are modest; for example, for $M=7, N=2$, the ODE integration took $\sim 0.2 \mathrm{cpu}$ s using a dual-core, AMD Opteron processor, model 175 , with $1 \mathrm{MB}$ cache, running at $2.2 \mathrm{GHz}$ with a 64 bit kernel.

The covariant Fourier harmonics of the field are saved at equally spaced radial intervals (with an arbitrarily fine radial mesh, $N_{i}$ ), and are then interpolated radially using cubic splines to give a continuous numerical representation of the field. An exactly divergence-free, to machine error, representation of the Beltrami field is achieved by calculating the field as the curl of the vector potential, $\mathbf{B}=\nabla \times(\mathbf{B} / \lambda)$.

A measure of the error in the solution is provided by the root mean square error quantities, $|\delta \dot{s}|$ $=\sqrt{\sum_{i, j, k}\left(\dot{s}_{g i j}-\dot{s}_{\nabla \times}\right)^{2} / N_{i} N_{j} N_{k}}, \quad$ and $\quad|\dot{\theta}|$ $=\sqrt{\sum_{i, j, k}\left(\dot{\theta}_{g i j}-\dot{\theta}_{\nabla \times}\right)^{2} / N_{i} N_{j} N_{k}}$, which are given as functions of the Fourier resolution, Fig. 4, for the configuration to be described in the following section. Here, $\dot{s}=B^{s} / B^{\zeta}$ and $\dot{\theta}$ $=B^{\theta} / B^{\zeta}$, and $\dot{s}_{g i j}, \dot{\theta}_{g i j}$ are calculated from "raising," and $\dot{s}_{\nabla \times}$, $\dot{\theta}_{\nabla \times}$ are calculated from the curl of the covariant components of the field. The sum $i, j, k$ is over the radial, poloidal and toroidal grid points. It is $\dot{s}$ and $\dot{\theta}$ which are required for the field line following the analysis presented in the following section.

\section{FIELD-LINE CHAOS}

For illustration, we consider a large-aspect-ratio, nearaxisymmetric configuration. The outer interface is a perturbation to a circular cross section, axisymmetric boundary of major radius $R_{0}$, minor radius $r_{1}, R=R_{0}+r_{1} \cos (\theta)$ and $Z$ $=r_{1} \sin (\theta)$, where $R_{0}=10.0$. Nontrivial solutions are obtained by imposing helical perturbations in the minor radius $r_{1}$ $=r_{1,0}-\delta \cos (2 \theta-\zeta) / 2-\delta \cos (3 \theta-\zeta) / 3, \quad$ where $\quad r_{1,0}=0.10$ with the single perturbation parameter, $\delta$, to be varied. The inner interface is chosen to be axisymmetric: $R=R_{0}$ $+r_{0,0} \cos (\theta)$ and $Z=r_{0,0} \sin (\theta)$, where $r_{0,0}=0.05$.

The boundary rotational transforms are controlled by the two degrees of freedom: the pitch-parameter, $\lambda$, and the ratio $I / G$. As far as the construction of a Beltrami field between two arbitrary interfaces is concerned, any selection of $\lambda$ and $I / G$ may be considered; however, we are primarily concerned with the case where the rotational-transforms on the inner, $t_{1}$, and outer, $t_{2}$, interfaces are constrained. There is sufficient freedom to achieve this, and a wide range of transform profiles are possible, as illustrated in Fig. 5 for the integrable case.

Taking the domain to be a subregion of the multiinterface system described in Secs. I and II, we take the rotational transforms at each interface to be the noble irrationals $t_{1}=0.177998 \ldots$ and $t_{2}=0.5607086 \ldots$. A simple numeri-
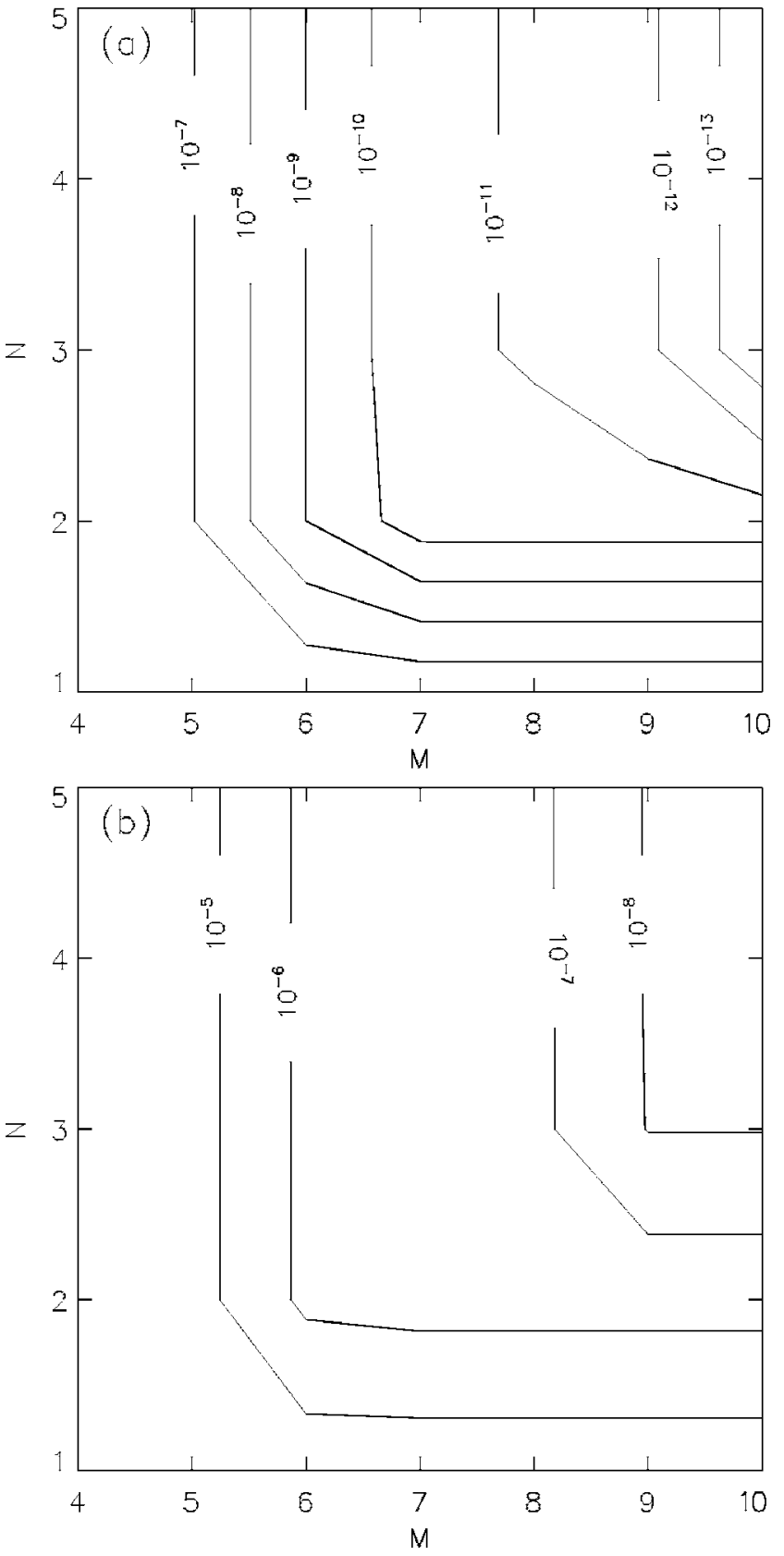

FIG. 4. Fourier convergence error: (a) $|\delta \dot{s}|$, and (b) $|\dot{\delta} \dot{\theta}|$, for the configuration with $\delta=0.0015$.

cal search determines the required pitch-parameter, $\lambda$ $=0.1376537$, and current ratio, $I / G=0.44502 \times 10^{-5}$ (for the integrable case $\delta=0.0$ ). The rotational-transform profile thus obtained is shown as the dotted line in Fig. 5. Note that the $t=\frac{1}{3}, \frac{1}{2}$ rational surfaces are present within this annular domain.

In the context of the (Hamiltonian) magnetic field, $\delta$ represents a chaos-inducing perturbation. For $\delta=0.0$, the system is toroidally symmetric and admits an integrable solution for the magnetic field; that is, the magnetic field lines lie on a continuous set of nested toroidal surfaces. For nonzero $\delta$, the helical perturbations result in primary magnetic islands at the $t=\frac{1}{3}, \frac{1}{2}$ rational surfaces. Additional, secondary islands will form at the $t=\frac{2}{5}, \frac{3}{8}, \frac{3}{7}, \ldots$ rational surfaces, but if $\delta$ is 


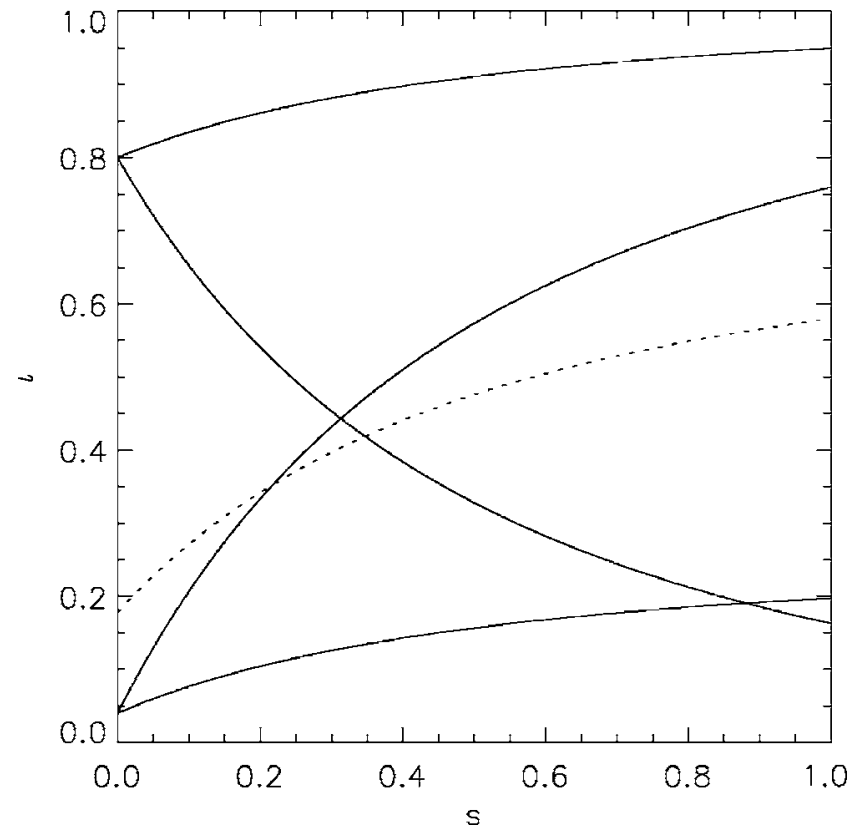

FIG. 5. Rotational transform profiles for $(\lambda, I / G)=\left(-0.01,2.0 \times 10^{-5}\right)$, $\left(0.20,2.0 \times 10^{-5}\right), \quad\left(0.05,0.1 \times 10^{-5}\right), \quad\left(0.20,0.1 \times 10^{-5}\right) \quad($ solid $), \quad$ and $\left(0.1376537,0.44502 \times 10^{-5}\right)$ (dotted) in the integrable case $\delta=0$. The rotational transform on the inner surface is $t=0.80,0.04$ for $I / G=2.0$ $\times 10^{-5}, 0.1 \times 10^{-5}$, and the greater the value of $\lambda$, the greater the shear.

small then these islands are small and some irrational surfaces between the $t=\frac{1}{3}, \frac{1}{2}$ rational surfaces will be present. Such a case is shown in Fig. 6, for $\delta=0.0015$. If $\delta$ is sufficiently large, the unstable manifolds associated with the $t=\frac{1}{3}, \frac{1}{2}$ islands will overlap and produce a region of connected chaotic field, as is shown in Fig. 7, for $\delta=0.0030$.

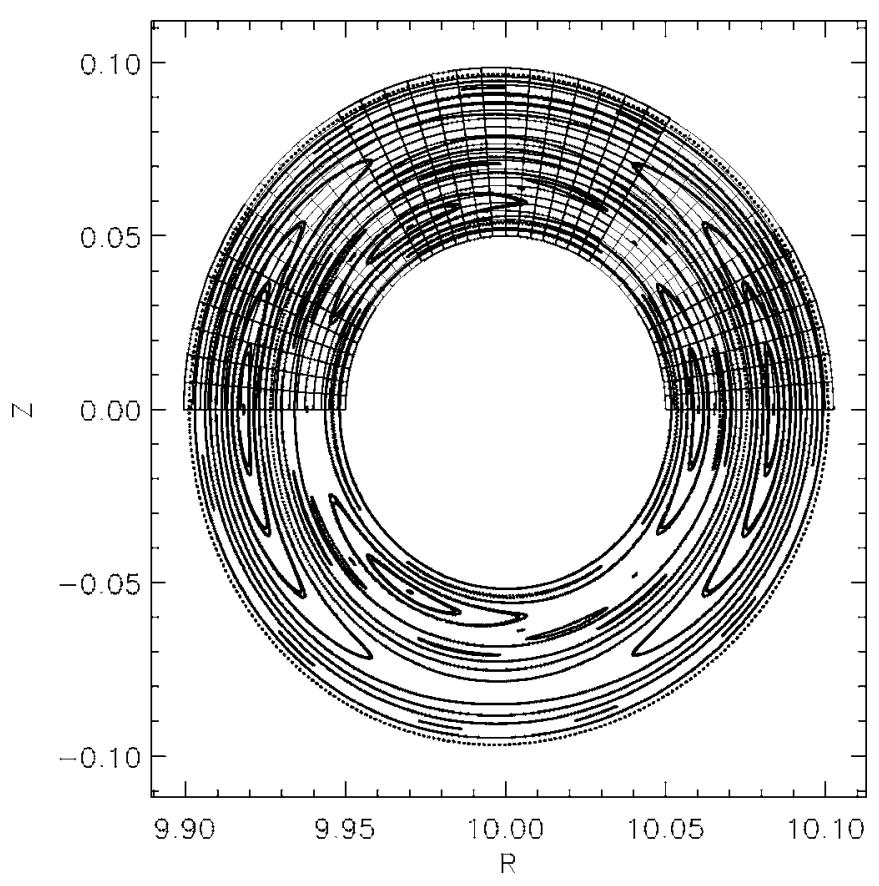

FIG. 6. Poincaré plot of the Beltrami field: perturbation of outer boundary $\delta=0.0015$, with Fourier resolution $M=7, N=2$. Shown in the upper half plot is the coordinates.

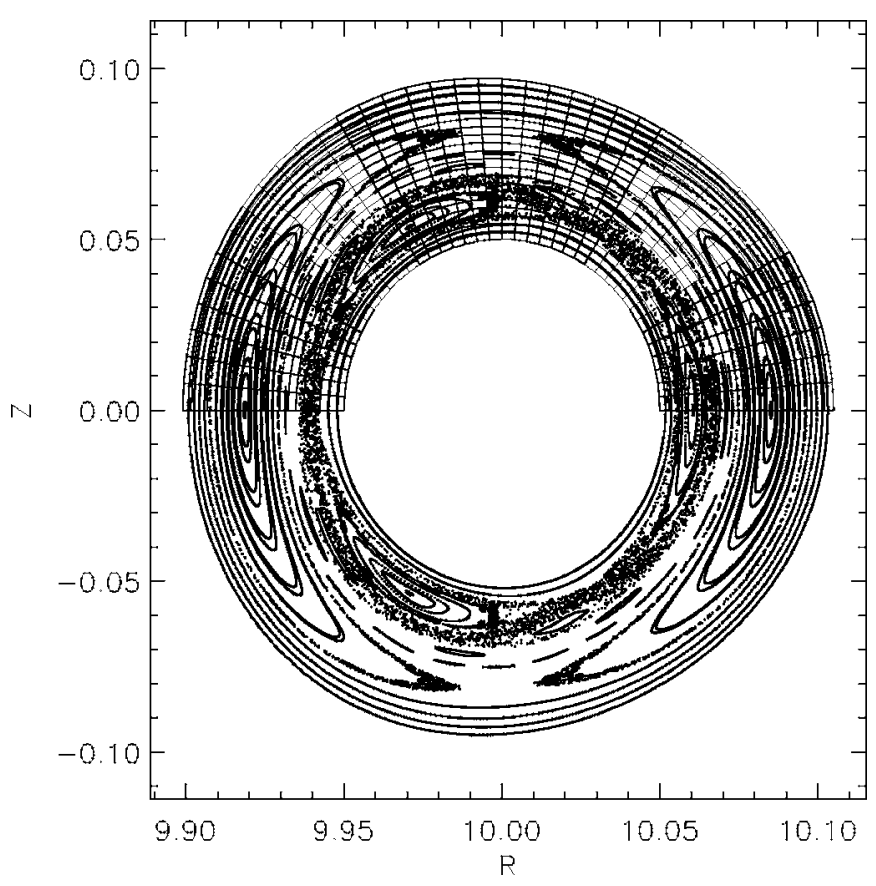

FIG. 7. Poincaré plot of Beltrami field: perturbation of outer boundary $\delta$ $=0.0030$, with Fourier resolution $M=7, N=2$. Shown in the upper half plot is the coordinates.

Note that, at fixed $\lambda$ and $I / G$, the rotational-transform at the outer interface, $t_{2}$, varies slightly with $\delta$ (less than 5 $\times 10^{-4}$ for $\delta=0.0030$, which corresponds to about a $0.2-0.3 \mathrm{~cm}$ deformation in the $10 \mathrm{~cm}$ minor radius).

Poincaré plots provided a simple graphical illustration of the degree of field-line chaos; however, more sophisticated approaches that are both more numerically efficient and give detailed information regarding the existence (or otherwise) of KAM surfaces are available, namely Greene's residue criterion $^{10,14}$ and its extensions. ${ }^{12,15}$ These approaches have been applied to magnetic field-line chaos in an earlier publication, ${ }^{47}$ and here only brief, intuitive remarks regarding the application of the residue criterion will be given.

The residue, $R$, is defined on the periodic orbits $R$ $=R(p, q)$, where $t=p / q$. In essence, it provides an indication of the size of the associated island chain (more precisely, it is related to the stability of an island chain, as determined by the eigenvalues of the tangent $\left.\operatorname{map}^{10}\right)$. A given irrational, KAM surface may be approximated arbitrarily closely by high order periodic orbits, and if the high-order island chains overlap then any enclosed KAM surfaces will be destroyed. Thus, by calculating the residue for a sequence of periodic orbits that successively better approximate a given irrational (such a sequence is provided by truncations of the continuedfraction representation ${ }^{13}$ ), the existence of the KAM surface may be determined. If the residues approach zero, the KAM surface exists; if the residues become large, the KAM surface has been destroyed; and if the residues approach 0.25 , the KAM surface is on the edge of destruction.

Here, a variant ${ }^{12,15}$ of the residue criterion is employed that may be more suitable from an algorithmic perspective. In the context of inserting additional interfaces, a necessary step toward the construction of a multiple-interface global- 


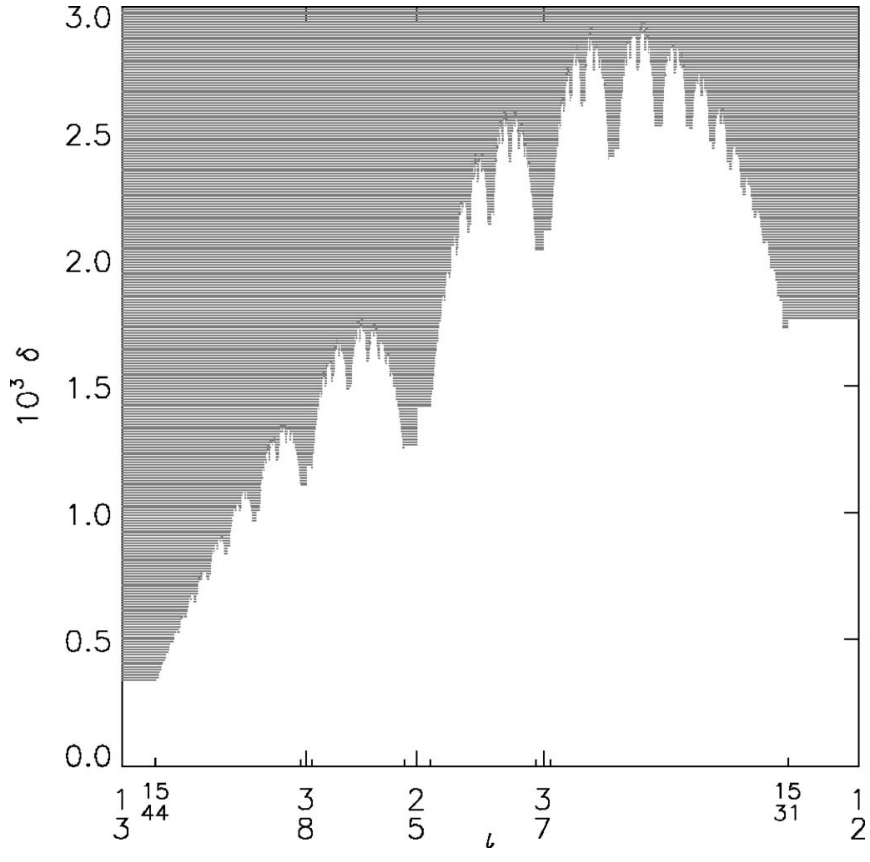

FIG. 8. Critical function for the family of Beltrami fields parametrized by the 3D perturbation parameter $\delta$, with Fourier resolution $M=7, N=2$, showing some low order rationals and their adjacent Farey intervals, to level 14. The gray areas indicate regions of connected chaos.

equilibrium code with an arbitrarily fine stepped-pressure profile, the first question that arises is: Do any KAM surfaces exist in a given region? Consider a pair of rationals, $\left(p_{1}, q_{1}\right)$ and $\left(p_{2}, q_{2}\right)$, that are neighboring, $\left|p_{1} q_{2}-p_{2} q_{1}\right|=1$. If the residues of these rational orbits are large compared to 0.25 , say $R\left(p_{1}, q_{1}\right)+R\left(p_{2}, q_{2}\right)>0.50$, then it is likely that all KAM surfaces in the region $\boldsymbol{t} \in\left[p_{1} / q_{1}, p_{2} / q_{2}\right]$ are destroyed. If, however, $R\left(p_{1}, q_{1}\right)+R\left(p_{2}, q_{2}\right)<0.50$, then there is a chance that at least one KAM surface exists. This method is reminiscent of the island overlap criterion; ${ }^{48}$ however, the "width" of an island with a chaotic separatrix is not so easily determined, whereas the residue is an easily calculated numerical quantity. The initial interval $\left[p_{1} / q_{1}, p_{2} / q_{2}\right]$ may be subdivided using the mediant, $\left(p_{1}+p_{2}, q_{1}+q_{2}\right)$ (and in such a manner the Farey tree of rationals is constructed $\left.{ }^{9}\right)$, and by testing the successive subintervals, an accurate method for identifying which KAM surfaces exist is enabled.

This method is applied to the case at hand, Fig. 8. For a given perturbation $\delta$, and beginning with $\left(p_{1}, q_{1}\right)=(1,3)$ and $\left(p_{2}, q_{2}\right)=(1,2)$, the residues for periodic orbits up to the 14th level of the Farey tree are constructed [with the exception that if $R\left(p_{i}, q_{i}\right)+R\left(p_{j}, q_{j}\right)>1.00$ then this region of field is deemed strongly chaotic and no attempt to further subdivide the interval is pursued]. Periodic orbits, and the residues, are located by field-line tracing ${ }^{47}$ (for locating periodic orbits in strongly chaotic regions, such as those that approximate the "broken-KAM" surfaces, i.e. the cantori, it is however preferable to use Lagrangian variational integration methods ${ }^{49}$ ).

For the case of stellarator-symmetric geometries, ${ }^{46}$ such as that considered here, all the required periodic orbits lie in symmetry lines. ${ }^{50} \mathrm{~A}$ symmetry line in this case is given by the line $\{(s, \theta, \zeta): \theta=0, \zeta=0\}$. This enables various numerical simplifications; for example, in principle, the twodimensional search for periodic orbits becomes a much simpler one-dimensional search along the symmetry line (see for example Refs. 47 and 49 , and the references therein).

If $R\left(p_{i}, q_{i}\right)+R\left(p_{j}, q_{j}\right)>0.5$, where $\left(p_{i}, q_{i}\right),\left(p_{j}, q_{j}\right)$ refer to adjacent rationals of the 14th Farey level, a gray horizontal line is plotted between $t=p_{i} / q_{i}$ and $t=p_{j} / q_{j}$, at vertical level $\delta$. Thus the gray area in Fig. 8, which is essentially the critical function, ${ }^{51}$ indicates regions of connected chaos.

Also shown in Fig. 8 are some low order rationals and the extent of the adjacent Farey intervals: for example, the interval adjacent to the $1 / 2$ rational is $[15 / 31,1 / 2]$, and this determines the resolution, in terms of the rotationaltransform, of the figure. The width of each Farey interval is determined by its "path" down the Farey tree, and alternating paths both produce smaller intervals and bound noble irrationals. The minimum Farey interval, at the 14th level, is [987/2584, 610/1597].

Note that the white areas in Fig. 8 do not necessarily indicate regions foliated with magnetic flux surfaces, merely that at least one KAM surface is likely to exist. The most robust KAM surface in a given region is typically that with the most irrational transform, ${ }^{12,15}$ though this ultimately depends on the perturbation spectrum imposed. Having thus identified the most likely candidates, the KAM surfaces can then be numerically determined. ${ }^{47}$

\section{DISCUSSION}

How to extend this construction of the Beltrami field in a single volume to a multiple-interface global equilibrium code remains the topic of ongoing investigation. We have already shown that reasonably realistic tokamak equilibria can be constructed within the cylindrical approximation. ${ }^{43}$ Further extension requires a number of issues to be resolved, and some speculation on these issues is given in the following.

One issue that needs to be considered is whether the rotational transform should be allowed to jump across a KAM barrier surface; whether it is one double-sided KAM surface or two single-sided KAM surfaces back-to-back. Rotational discontinuities are allowed in ideal MHD and are observed in the solar wind. ${ }^{52}$ They imply the existence of current sheets, but so do any surfaces with pressure jumpsthis is not an argument against rotational transform jumps. A preliminary investigation of this has already been performed in cylindrical geometry, ${ }^{44}$ where a single interface $S_{i}$, with a jump in $t$, is modelled by two interfaces, with no $t$ jump, in the limit that the two interfaces approach each other.

The single interface model described above can be extended to a multiple interface model by including additional interfaces, chosen, before establishing a pressure difference, to coincide with the existing KAM surfaces. Consider inserting an additional flux barrier between the two originally prescribed interfaces $S_{i-1}$ and $S_{i}$. For example, let this additional interface, denoted by $S_{i-1 / 2}$ be inserted between the $t=\frac{1}{3}, \frac{1}{2}$ island chains, which for the sake of argument are assumed not to overlap, so that at least one surviving KAM surface exists in this region. Let $V_{i-1 / 2}$ be the volume enclosed by, and including, the interfaces $S_{i-1}$ and $S_{i-1 / 2}$. 
With no further work, such a KAM surface may already be considered as an additional flux barrier-a trivial interface with no pressure jump. However, when a pressure jump $\delta p_{i-1 / 2}$, is introduced at the $S_{i-1 / 2}$ interface, by increasing the entropy in the new subregion $V_{i-1 / 2}$, the flux barrier will have to move to resolve the unbalanced forces across the interface [or, equivalently, to minimize the free energy $F$, Eq. (1)]. This will in general alter the rotational transforms at the boundaries of the subdomains $V_{i-1 / 2}$ and $V_{i}$, but we have shown in this paper that they can be returned to their initial values by adjusting (at the expense of conservation of helicity and poloidal flux) the eigenvalues $\lambda_{i-1 / 2}$ and $\lambda_{i}$, thus restoring the flux barrier to a KAM barrier. The consequent changes in $\mathbf{B}$ will mean that force balance is again violated, and thus the process must be repeated iteratively until both force balance and the irrational transform requirements are simultaneously satisfied (to within specified tolerances).

Presumably, for a good plasma confinement configuration and a sufficiently small pressure jump $\delta p_{i+1 / 2}$, the iteration will converge. However, at some threshold value, one surmises that the KAM barrier will lose its smoothness in a similar fractal manner to the known behavior of critical KAM surfaces, ${ }^{12}$ and beyond this point it will no longer be possible to find a solution. In future work, we will consider these issues in more detail, in particular examining further whether the number theory requirements on the rotational transforms can be built into a variational principle.

The present paper has used a simple, straightforward approach to solving the Beltrami equation in an arbitrary toroidal region, which will provide a benchmark with which to compare more sophisticated approaches. Two other approaches spring naturally to mind: Galerkin methods exploiting the variational nature of the problem (cf. VMEC, Ref. 16) and mesh-free boundary integral/Green's function methods $^{38,39,53-55}$ exploiting the linearity of the Beltrami equation. Which numerical treatment is ultimately most suitable for solving this local, single-interface problem, must however be understood in the context of the overall efficiency and robustness of a numerical code that addresses the global, multiple-interface problem.

A further issue to be examined is resolving the sheet current singularities at our zero-width flux barriers by thickening them into thin shells of plasma described by ideal MHD, in which $\nabla p$ is finite (except in the limit as the width goes to zero). A KAM barrier can be modeled by assuming there is no magnetic shear within the shell, so the irrational rotational transform remains constant across it. Then we can adjust the $\lambda$ 's in the neighboring regions until the boundary rotational transforms in the adjacent Beltrami fields match that in the ideal region. With no resonant regions in the shell, a self-consistent equilibrium based on continuously nested magnetic surfaces should be straightforward to construct, and could be tested for interchange (Mercier) and ballooning stability in a similar spirit to the approach of Hegna and Nakajima ${ }^{56}$ (although the vanishing of the magnetic shear in the ballooning equation means there are no secular terms, the quasiperiodicity of the coefficients in the ballooning equation can lead to Anderson localization ${ }^{57}$ ).

A finite-width barrier with a rotational transform jump across it is more problematical as it necessarily has magnetic shear and thus will contain rational surfaces where the problems referred to in Sec. I will occur. An approach to resolving the issue of whether rotational-transform jumps are allowed in 3D is to construct such a finite-width barrier as a multi-interface Beltrami equilibrium in microcosm. A beginning at this approach has been attempted in Ref. 44.

\section{ACKNOWLEDGMENTS}

We thank Allen Boozer, Don Monticello, Neil Pomphrey, Phil Morrison, and Robert MacKay for stimulating discussions.

This work was supported in part by the U.S. Department of Energy Contract No. DE-AC02-76CH03073 and Grant No. DE-FG02-99ER54546 and the Australian Research Council.

${ }^{1}$ A. H. Boozer, Phys. Fluids 26, 1288 (1983).

${ }^{2}$ J. R. Cary and R. G. Littlejohn, Ann. Phys. (Paris) 151, 1 (1983).

${ }^{3}$ W. D. D'haeseleer, W. N. G. Hitchon, J. D. Callen, and J. L. Shohet, Flux Coordinates and Magnetic Field Structure (Springer, Berlin, 1991), p. 170.

${ }^{4}$ R. L. Dewar, D. A. Monticello, and W. N.-C. Sy, Phys. Fluids 27, 1723 (1984).

${ }^{5}$ H. Goldstein, Classical Mechanics, 2nd ed. (Addison-Wesley, Massachusetts, 1980), Chap. 10.

${ }^{6}$ A. H. Boozer, Phys. Plasmas 5, 1647 (1998).

${ }^{7}$ D. K. Arrowsmith and C. M. Place, An Introduction to Dynamical Systems (Cambridge University Press, Cambridge, U. K., 1991).

${ }^{8}$ A. J. Lichtenberg and M. A. Lieberman, Regular and Chaotic Dynamics, 2nd ed. (Springer-Verlag, New York, 1992).

${ }^{9}$ J. D. Meiss, Rev. Mod. Phys. 64, 795 (1992).

${ }^{10}$ J. M. Greene, J. Math. Phys. 20, 1183 (1979).

${ }^{11}$ S. R. Hudson, D. A. Monticello, A. H. Reiman, A. H. Boozer, D. J. Strickler, S. P. Hirshman, and M. C. Zarnstorff, Phys. Rev. Lett. 89, 275003 (2002).

${ }^{12}$ R. S. MacKay and J. Stark, Nonlinearity 5, 867 (1992).

${ }^{13}$ I. Niven, Irrational Numbers, Mathematical Association of America (Wiley, New York, 1956).

${ }^{14}$ R. S. MacKay, Nonlinearity 5, 161 (1992).

${ }^{15}$ J. M. Greene, R. S. MacKay, and J. Stark, Physica D 21, 267 (1986).

${ }^{16}$ S. P. Hirshman and Whitson, Phys. Fluids 26, 3553 (1983).

${ }^{17}$ H. J. Gardner and D. B. Blackwell, Nucl. Fusion 32, 2009 (1992).

${ }^{18}$ A. H. Boozer, Phys. Fluids 24, 1999 (1981).

${ }^{19}$ A. Bhattacharjee, T. Hayashi, C. C. Hegna, N. Nakajima, and T. Sato, Phys. Plasmas 2, 883 (1995).

${ }^{20}$ H. Grad, Phys. Fluids 10, 137 (1967).

${ }^{21}$ A. H. Reiman and H. S. Greenside, J. Comput. Phys. 75, 423 (1988).

${ }^{22}$ K. Harafuji, T. Hayashi, and T. Sato, J. Comput. Phys. 81, 169 (1989).

${ }^{23}$ M. D. Kruskal and R. M. Kulsrud, Phys. Fluids 1, 265 (1958).

${ }^{24}$ J. B. Taylor, Phys. Rev. Lett. 33, 1139 (1974).

${ }^{25}$ J. B. Taylor, Rev. Mod. Phys. 58, 741 (1986).

${ }^{26}$ J. H. Misguich, Phys. Plasmas 8, 2132 (2001).

${ }^{27}$ J. H. Misguich, J.-D. Reuss, D. Constantinescu, G. Steinbrecher, M. Vlad, F. Spineanu, B. Weyssow, and R. Balescu, Ann. Phys. (Paris) 28, 1 (2003).

${ }^{28}$ O. P. Bruno and P. Laurence, Commun. Pure Appl. Math. 49, 1717 (1996).

${ }^{29}$ H. L. Berk, J. P. Freidberg, X. Llobet, P. J. Morrison, and J. A. Tataronis, Phys. Fluids 29, 3281 (1986)

${ }^{30}$ R. Kaiser and A. Salat, Phys. Plasmas 1, 281 (1994).

${ }^{31}$ R. Kaiser, Phys. Plasmas 1, 2693 (1994).

${ }^{32}$ G. O. Spies and J. Li, Phys. Plasmas 1, 2901 (1994).

${ }^{33}$ G. O. Spies, D. Lortz, and R. Kaiser, Phys. Plasmas 8, 3652 (2001).

${ }^{34}$ G. O. Spies, Phys. Plasmas 10, 3030 (2003).

${ }^{35}$ A. Bhattacharjee and R. L. Dewar, Phys. Fluids 25, 887 (1982).

${ }^{36}$ L. Woltjer, Astrophys. J. 128, 384 (1958).

${ }^{37}$ P. J. Morrison, Rev. Mod. Phys. 70, 467 (1998), p. 511

${ }^{38}$ R. Kress, Z. Angew. Math. Phys. 28, 715 (1977).

${ }^{39}$ R. Kress, J. Eng. Math. 15, 29 (1981). 
${ }^{40}$ R. Kress, J. Eng. Math. 20, 323 (1986).

${ }^{41}$ D. Lortz and G. O. Spies, Phys. Plasmas 1, 249 (1994).

${ }^{42}$ R. Kaiser and H. Uecker, Q. J. Mech. Appl. Math. 57, 1 (2004).

${ }^{43}$ M. J. Hole, S. R. Hudson, and R. L. Dewar, J. Plasma Phys. 72, 1167 (2006).

${ }^{44}$ M. J. Hole, S. R. Hudson, and R. L. Dewar, "Equilibria and stability in partially relaxed plasma-vacuum systems," Nucl. Fusion (to be published). ${ }^{45} \mathrm{~S}$. Wolfram, The Mathematica Book, 5th ed. (Wolfram Media, Champaign, 2003), pp. 780-781.

${ }^{46}$ R. L. Dewar and S. R. Hudson, Physica D 112, 275 (1997).

${ }^{47}$ S. R. Hudson, Phys. Plasmas 11, 677 (2004).
${ }^{48}$ B. Chirikov, Phys. Rep. 52, 263 (1979).

${ }^{49}$ S. R. Hudson, Phys. Rev. E 74, 056203 (2006).

${ }^{50}$ H. Kook and J. D. Meiss, Physica D 1-2, 65 (1989).

${ }^{51}$ S. Marmi and J. Stark, Nonlinearity 5, 743 (1992).

${ }^{52}$ J. W. Belcher and L. Davis, Jr., J. Geophys. Res. 76, 3534 (1971).

${ }^{53}$ O. P. Bruno and F. Reitich, J. Fourier Anal. Appl. 7, 169 (2001).

${ }^{54}$ S. P. Hirshman, W. I. van Rij, and P. Merkel, Comput. Phys. Commun. 43, 143 (1986).

${ }^{55}$ P. Merkel, J. Comput. Phys. 66, 83 (1986).

${ }^{56}$ C. C. Hegna and N. Nakajima, Phys. Plasmas 5, 1336 (1998).

${ }^{57}$ P. Cuthbert and R. L. Dewar, Phys. Plasmas 7, 2302 (2000). 